\title{
Actitud de los padres frente al comportamiento de sus hijos hiperactivos
}

\author{
Mendoza-Reyes Patricia ${ }^{1}$, Montesinos-Matos Patricia ${ }^{1}$, Valderrama-Mendoza Vanessa ${ }^{1}$, \\ Silva- Mathews Zulema
}

\section{RESUMEN}

Objetivo: identificar la actitud de los padres frente al comportamiento de sus hijos hiperactivos escolares que acuden al Centro Educativo Básico Especial «Los Pinos»- San Juan de Lurigancho, en el año 2009. Material y métodos: estudio de diseño descriptivo de corte trasversal realizado con 41 padres a quienes se les aplicó un test de actitud frente al comportamiento de su hijo hiperactivo. Resultados: el 58,5\% de los padres encuestados mostró una actitud buena, mientras que el $41,5 \%$ presentó una actitud regular. En relación al componente cognitivo de la actitud el $44,4 \%$ de los padres presentó un nivel bueno y el 55,5\% un nivel regular; el 43,47 de las madres mostró un nivel regular y un 56,5 un nivel bueno En el componente afectivo de la actitud, los padres presentaron en un $50 \%$ un nivel tanto regular como bueno; en tanto las madres, el 73,91 un nivel regular y un 26,09 un nivel bueno. En el componente conductual de la actitud el $61,11 \%$ de los padres presentó una actitud buena, el 22,22\% regular y el $16,6 \%$ mala. En tanto, las madres en un $86,95 \%$ presentaron una actitud buena, el 8,69 regular y el 4,34 mala. Conclusiones: más de la mitad de los padres tienen una actitud buena frente al comportamiento del niño hiperactivo, según el componente cognitivo de la actitud los padres en su mayoría cuentan con conocimientos aplicados en el manejo de su hijo. En relación al componente afectivo, en su mayoría los padres tienen una respuesta afectiva regular. En tanto en el componente conductual la respuesta de los padres fue buena.

Palabras clave: Trastorno de déficit de atención; Hiperactividad; Actitudes y padres. Perú. (Fuente DeCs BIREME).

\section{Attitude of the parents opposite to the behavior of his hyperactive children}

\begin{abstract}
Objetive: To identify the attitude of the parents opposite to the behavior of his hyperactive school children who come to the Educational Basic Special Center " The Pines " - Lurigancho's San Juan, in the year 2009. Material and methods: study of descriptive design of court trasversal realized with 41 parents to whom a test of attitude was applied opposite to the behavior of his hyperactive son. Material and Methods: Study of descriptive design of court trasversal realized with 41 parents to whom a test of attitude was applied opposite to the behavior of his hyperactive son. Results: $58,5 \%$ of the polled parents showed a good attitude, whereas $41,5 \%$ presented a regular attitude. In relation to the cognitive component of the attitude $44,4 \%$ of the parents presented a good level and 55,5\% a regular level; 43,47 of the mothers it showed a regular level and 56,5 a level good At the affective component of the attitude, the parents presented in 50 $\%$ a both regular and good level; while the mothers, 73, 91 a regular level and 26,09 a good level. In the behavioral component of the attitude $61,11 \%$ of the parents presented a good attitude, $22,22 \%$ to regulate and $16,6 \%$ bad. While, the mothers in 86,95\% presented a good attitude, 8,69 regular and 4,34 bad. Conclusions. More than the half of the parents they have a good attitude opposite to the behavior of the hyperactive child, according to the cognitive component of the attitude the parents in the main possess knowledge applied in the managing of his son. In relation to the affective component, in the main the parents have an affective regular response. While at the behavioral component the response of the parents was good.
\end{abstract}

Key words: Attention deficit hyperactivity disorder; Father; Attitudes. Peru. (Source DeCs BIREME).

Estudiante de Enfermería de la Facultad de Enfermería de la Universidad Peruana Cayetano Heredia.

Magister en Enfermería. Docente de la Facultad de Enfermería de la Universidad Peruana Cayetano Heredia. 


\section{INTRODUCCIÓN}

La incidencia de la hiperactividad en el Perú ocurre en el 5 al 10\% de los niños en edad escolar, y en el 3 al $6 \%$ de los adultos, constituyendo el mayor número de pacientes que trata un neurólogo o psiquiatra de niños y adolescentes en los consultorios externos (1).

La hiperactividad es la alteración del comportamiento de origen genético que se debe a un desequilibrio bioquímico cerebral, caracterizado por la presencia de tres síntomas principales: la falta de atención, hiperactividad e impulsividad, o de ambos; es un trastorno crónico y estos síntomas pueden variar de una etapa de vida a otra (1).

La actitud es un estado de disposición psicológica adquirida y organizada a través de la propia experiencia que incita al individuo a reaccionar de una manera específica frente a determinadas personas, objetos y situaciones, la actitud está organizada por una estructura: el componente cognitivo, que implica el conocimiento, creencias, valores y pensamientos; el componente afectivo, que comprende las emociones positivas o negativas frente a una situación, es un sentimiento o carga afectiva que se deposita sobre una determinada situación; y el componente conductual, que consiste en la predisposición o intención para actuar en forma especifica que sea relevante a la actitud $(2,3)$.

La actitud que presenten los padres ante su hijo hiperactivo marcará la diferencia en el desarrollo futuro de este. Se debe precisar que los padres deben comprender el trastorno, la impulsividad, la facilidad para despistarse, el incumplimiento de las órdenes, y la necesidad que se establezcan metas simples y concretas a corto plazo, considerando que su hijo olvida fácilmente. Muchas veces los padres reaccionan con expresiones tales como «este niño me agota», «es que no para», «i es que nunca se te acaban las pilas?» Es necesario señalar que lo que puede ser motivo de crisis para una familia no lo sea para otra, todo depende de la actitud de los padres para enfrentar a esta situación (4).

El entorno que rodea al niño hiperactivo, que no cuenta con el conocimiento de cómo tratarlos, asumiendo mitos y creencias sobre el comportamiento que tienen estos niños, ocasionará en ellos que se aíslen, tengan una baja autoestima, etc. (4).

Los padres, en todas las sociedades, son los agentes primarios para promover el desarrollo del niño, las actitudes de los padres tienen sus aspectos positivos y negativos, por lo que pueden adoptar conductas de hostilidad o aceptación hacia su hijo hiperactivo; estas actitudes de sobreprotección, de sobre indulgencia, o de rechazo son consideradas actitudes inadecuadas, y se manifiestan tanto en las madres como en los padres (5).

Tener un hijo hiperactivo es una de las tareas más difíciles para cualquier padre; personas que se sentían competentes y seguras en su vida adulta de pronto descubren que no pueden entender el comportamiento de su hijo. El conocer que su niño presenta un trastorno crónico los afecta profundamente, presentando muchas veces un conflicto de sus sentimientos. Estos niños hiperactivos son inquietos, desorganizados, perturban el salón de clases, no pueden mantenerse sentados por largo tiempo, no aprenden de la experiencia o la enseñanza, tienen bajo rendimiento escolar; frecuentemente se olvidan de las tareas y los maestros lo consideran ociosos, desafiantes, Presentando comportamientos que mayormente no se dirigen a las reglas (6).

El niño hiperactivo pone en tensiones y desorganiza no solo a los padres sino a la familia entera; es por ello que no se retira la idea de los autores, de que, a veces los castigos severos por parte de los padres son más frecuentes, lo que afecta ciertamente al niño y continúa con su hiperactividad. La mayoría de los padres no sabe qué hacer en estos casos, sin indagar mucho se deciden por castigos físicos y psicológicos hacia sus hijos (7).

Debido a estos problemas de salud mental el niño hiperactivo puede ser visto por la familia como un ente extraño a ella. Los padres con hijos hiperactivos desarrollan un aislamiento peculiar, se apartan, pero mantienen un intercambio material y emocional con el centro especializado en el manejo de niños hiperactivos $(8-9)$.

En nuestra experiencia, dentro de las prácticas hospitalarias, se encontró casos en los cuales los padres con niños hiperactivos reflejaban sentimientos positivos pues acudían al hospital a dejar a sus hijos en los talleres o para consulta con el neurólogo, o el psiquiatra infantil. A los niños hiperactivos se les brindaba mucha atención, pero muchas veces el personal de salud no brinda a los padres el apoyo físico y emocional que requieren, es por ello que se observa en ellos signos de tristeza, cansancio, y expresando emociones y sentimientos negativos.

La enfermera tiene un papel relevante en su intervención con el niño hiperactivo; de ahí la importancia que brinde apoyo a los padres con hijos hiperactivos, a través de intervenciones educativas que les permitan un adecuado 
manejo del comportamiento de sus hijos hiperactivos, favoreciendo la relación familiar, apoyando y fortaleciendo el desarrollo del niño; como también favoreciendo las actitudes positivas, y mejorando aquellas actitudes regulares de los padres.

El presente artículo tiene por objetivo determinar la actitud de los padres frente al comportamiento de sus hijos hiperactivos que acuden al Centro Educativo Básico Especial «Los Pinos»- San Juan de Lurigancho entre marzo y abril de 2009.

\section{MATERIAL Y MÉTODOS}

Investigación de tipo descriptiva de corte transversal, realizada en el Centro Educativo Básico especial en San Juan de Lurigancho. La población estuvo conformada por 41 padres de familia, que cumplieron con los criterios de selección, y participaron voluntariamente firmando el consentimiento informado.

La técnica utilizada para la recolección de la información fue la encuesta, y como instrumento se utilizó el test de actitud basada en la Escala de Likert, elaborada por las autoras, y que constó de 24 preguntas, estableciendo escalas de medición ordinal y por intervalos.

Cada enunciado presentaba cinco alternativas de respuesta: Totalmente de acuerdo (TA); De acuerdo (A); Indiferente (I); Desacuerdo (D), y Totalmente en desacuerdo (TD), con puntajes del 0 al 4 si el enunciado es negativo, $o$ de 4 al 0 si el enunciado es positivo.

La sumatoria de las puntuaciones obtenidas para cada enunciado indica la dirección de la actitud, el mayor puntaje direccionaba hacia una actitud buena; a menor puntaje una actitud mala. La prueba también comprendió de un puntaje mínimo dentro de la actitud de 0 , y un máximo de 96.

Se establecieron categorizaciones dentro de la variable actitud de los padres, en un rango de: Mala de 0 a 31 puntos; Regular de 32 a 63, y Buena de 64 a 96 puntos.

Asimismo se estableció puntos de corte en el componente cognitivo, afectivo y conductual de la actitud. En lo cognitivo, con un puntaje mínimo de 0 y máximo de 24 ; en lo afectivo un mínimo de 0 y máximo de 40 , y en lo conductual un mínimo 0 y máximo 32, considerando el rango de Mala, Regular y Buena.

El instrumento fue sometido a juicio de experto y a prueba piloto, se determinó los coeficientes de confiabilidad de mitades de Sperman-Brow y Gutman, también se cálculo el coeficiente de Alfa de Crombach teniendo como resultado 0,636 .

Para el análisis de los datos se aplicó la estadística descriptiva e inferencial, distribución de frecuencias para poder realizar el análisis de los resultados obtenidos.

En la investigación se respetaron los principios bioéticas y se contó con la aprobación del Comité Institucional de Ética de la Universidad Peruana Cayetano Heredia.

\section{RESULTADOS}

Tabla 1. Actitud de los padres frente al comportamiento de sus hijos hiperactivos que acuden al Centro Educativo Básico Especial «Los Pinos»-San Juan de Lurigancho.

Marzo - Abril 2009

\begin{tabular}{lcc}
\hline Actitud de los padres & N. $^{\circ}$ & \% \\
\hline Regular & 17 & 41,5 \\
Buena & 24 & 58,5 \\
Total & 41 & 100.0 \\
\hline
\end{tabular}

Tabla 2. Actitud de los padres en su componente cognitivo frente al comportamiento de sus hijos hiperactivos que acuden al Centro Educativo Básico Especial «Los Pinos»San Juan de Lurigancho. Marzo - Abril 2009

\begin{tabular}{lccrccc}
\hline \multirow{2}{*}{ Padres } & \multicolumn{6}{c}{ Cognitivo } \\
\cline { 2 - 7 } & \multicolumn{2}{c}{ Regular } & \multicolumn{2}{c}{ Buena } & \multicolumn{2}{c}{ Total } \\
\cline { 2 - 7 } & N. & $\%$ & N. ${ }^{\circ}$ & $\%$ & N. $^{\text {o }}$ & $\%$ \\
\hline Padre & 10 & 55,50 & 8 & 44,4 & 18 & 100 \\
Madre & 10 & 43,47 & 13 & 56,5 & 23 & 100 \\
\hline
\end{tabular}

Tabla 3. Actitud de los padres en su componente afectivo frente al comportamiento de sus hijos hiperactivos que acuden al Centro Educativo Básico Especial «Los Pinos»-

San Juan de Lurigancho. Marzo - Abril 2009

\begin{tabular}{|c|c|c|c|c|c|c|}
\hline \multirow{3}{*}{ Padres } & \multicolumn{6}{|c|}{ Afectivo } \\
\hline & \multicolumn{2}{|c|}{ Regular } & \multicolumn{2}{|c|}{ Buena } & \multicolumn{2}{|c|}{ Total } \\
\hline & N. & $\%$ & $\mathrm{~N}^{\circ}$ & $\%$ & $\mathrm{~N}^{\circ}{ }^{\circ}$ & $\%$ \\
\hline Padre & 9 & 50 & 9 & 50 & 18 & 100 \\
\hline Madre & 17 & 73,9 & 6 & 26,08 & 23 & 100 \\
\hline
\end{tabular}


Tabla 4. Actitud de los padres en su componente conductual frente al comportamiento de sus hijos hiperactivos que acuden al Centro Educativo Básico Especial «Los Pinos»San Juan de Lurigancho. Marzo - Abril 2009

\begin{tabular}{lcccccc}
\hline \multirow{2}{*}{ Padres } & \multicolumn{6}{c}{ Conductual } \\
\cline { 2 - 7 } & \multicolumn{2}{c}{ Regular } & \multicolumn{2}{c}{ Buena } & \multicolumn{2}{c}{ Total } \\
\cline { 2 - 7 } & N. & $\%$ & N. $^{\circ}$ & $\%$ & N. $^{\text {o }}$ & $\%$ \\
\hline Padre & 3 & 16,6 & 4 & 22,22 & 18 & 100 \\
Madre & 1 & 4,34 & 2 & 8,69 & 23 & 100 \\
\hline
\end{tabular}

\section{DISCUSIÓN}

El trastorno de hiperactividad es la alteración del comportamiento más común de la niñez, que se manifiesta por un nivel inusualmente elevado y crónico de falta de atención, impulsividad y sobreactividad, o de ambos a la vez; afecta a más de 2 millones de niños en edad escolar (1).

El los resultados se puede observar que el $41,5 \%$ de los padres participantes en el estudio presentaron una actitud regular, mientras que el 58,5\% una actitud buena (Tabla $1)$.

El convivir con un niño con déficit de atención suscita una mezcla de sentimientos, por un lado se reconocen sus valores y potencialidades pero, por otro lado, hay que reconocer que no siempre es fácil vivir con ellos, ya que con frecuencia surgen situaciones que dificultan la vida familiar, razón por la que a más información que el padre tenga sobre el comportamiento de su hijo hiperactivo, mejor será el desempeño y su actitud hacia ellos $(7,9)$.

La actitud buena que se ha encontrado en 24 padres puede estar relacionada con el rol de protección hacia sus hijos, de ello surge la sobreprotección, lo cual resulta adecuado, según su perspectiva. Por otro lado, actualmente la alianza que se establece entre las instituciones de apoyo y los padres permite que estos cuenten con una orientación sobre el manejo de su hijo con hiperactividad, contribuyendo a que la expectativa sea positiva conforme crezca el niño, se genera confianza y se crea un sentimiento de acompañamiento para afrontar la situación (10).

La Asociación Peruana de Déficit de Atención (APDA), constituida por padres de chicos con TDAH, emite boletines electrónicos trimestrales desde hace tres años y tiene una página web (11) con más de medio centenar de artículos escritos especialmente para el boletín y la web; también se dicta charlas y talleres para padres, para maestros $\mathrm{y}$ otros profesionales. Ha elaborado a través de dos abo- gados, padres de niños con TDAH, un proyecto de ley que ha sido modificado y presentado por una congresista; actualmente está en la Comisión de Salud del Congreso de la República (11).

Los padres son responsables del desarrollo del niño hiperactivo y de su mejoría, esta es una buena actitud que adoptan estos padres, a pesar de que el comportamiento y el manejo del niño hiperactivo resulta ser un reto, el cual es facilitado porque un gran porcentaje cuenta con formación educativa concluida lo cual favorece el entendimiento sobre las características, la sintomatología y el tratamiento de su hijo hiperactivo, es decir, se muestran más involucrados, desempeñando bien su rol paternal, para no causar daño a sus hijos buscando siempre su bienestar.

Las características y las conductas, tanto de la madre como del niño, pueden influir en la identidad del rol maternal y del niño. Las características maternas incluidas en el modelo de Mercer son la simpatía y la sensibilidad a las señales emitidas por el niño (12). Esto quiere decir que la interacción entre la madre y el niño, va a desarrollar sentimientos afectivos que rigen la actitud de la madre, siendo esta una actitud comprensiva, tolerante, lo que podemos entender como una actitud buena.

La aceptación global de un hijo ejerce un profundo impacto en el autoconcepto del padre y en su relación con lo demás; las actitudes paternas están influidas por la concepción que los adultos tienen de su papel en relación con los hijos, esta actitud puede estar influenciada por el comportamiento del niño, lo cual va a generar en el padre actitudes de protección, sentimientos positivos al querer mantener un bienestar en el niño en todo momento (13, 14).

Dado que la actitud de una persona está determinada por un componente cognitivo, afectivo y conductual (15), en la investigación se analizó el comportamiento de estos componentes en los padres encuestados, encontrando que el $44,4 \%$ (8) de los padres presentaban un componente cognitivo bueno, mientras que el 55,5\% (10) es regular, en cambio la actitud de las madres fue de 56,5 (13) bueno y 43,47 (10) fue regular; lo que implica que la racionalización que hacen de una idea para actuar sobre el comportamiento de su hijo hiperactivo es buena y regular respectivamente (Tabla 2).

Según Epictetus, sobre la teoría de la actitud, menciona que aprendemos actitudes del mismo modo en que aprendemos todo lo demás. Al aprender la información nueva, aprendemos sentimientos, pensamientos y las acciones que 
están en relación con ella. En la medida en que seamos recompensados (reforzados) por ellas, el aprendizaje perdurará (16).

En tanto algunos padres sintiéndose afectados por la presencia de un niño al que posiblemente no esperaban, desarrollarán mecanismos que le impidan buscar información, y se niegan a permanecer por mucho tiempo, solo lo necesario, con sus niños.

Mediante las experiencias previas, todos tenemos o adoptamos posiciones ante determinados hechos sociales, todos hemos aprendido a dar una respuesta valorativa cognitiva, estas respuestas previas se define como actitudes (16).

En los primeros momentos del proceso de enfrentarse ante determinada situación de una persona, en el entorno familiar es frecuente que se utilice la negación como un medio para controlar miedos y ansiedad. Así, es común encontrar a padres de familia que se nieguen a aceptar las evidencias que su hijo es hiperactivo, y que no se comporte igual que sus hermanos o compañeros de clase, y que necesite la ayuda de otras personas para mantener su adaptación al medio. Otra forma de negar el problema es evitar hablar del caso de su hijo hiperactivo (17).

Este estadio es, normalmente, temporal. Pero conforme este estadio continúe y no se maneje de manera adecuada para mantener su autonomía funcional se hacen más evidentes, empieza a hacerse cada vez más difícil creer que el paciente está «simplemente distraído» o que se trata de una «enfermedad temporal» (14).

Estas teorías del aprendizaje conciben a las personas como seres primariamente pasivos, cuyo aprendizaje depende del número y de la fuerza de los elementos positivos y negativos previamente aprendidos (18).

Según las teorías de la consistencia cognitiva, la incoherencia entre dos estados de conciencia hace que las personas se sientan incómodas. En consecuencia, cambian sus pensamientos o las acciones, el componente cognitivo de la actitud, está conformada por la información, que implica las creencias frente a la enfermedad, es decir la estructura vertical que significa que una creencia primaria afectará una creencia de menor importancia bajo ella (15).

Los mitos, valores, y pensamientos que tenemos acerca de hiperactividad, pueden ser adquiridas por algunas personas pertenecientes a generaciones anteriores que se dibujan como representantes de esos valores. Casi nunca son expresados con claridad como tales, pero pueden averiguarse a través de los valores mantenidos por la familia (1).

Los mitos familiares aquellas consignas o verdades incontestables que se transmiten de generación en generación, usualmente de una manera implícita, y que dotan de valores a los miembros de las familias, pueden influir en la actitud de los padres (16).

Las creencias, al igual que los valores, forman parte de nuestras actitudes y por ello rigen diversos fenómenos. Los grupos a los que pertenece una persona, tanto familiar como referida a las amistades, o los grupos secundarios que engloban las organizaciones o instituciones, pueden ser fuentes de confusión y tensión (14).

Se hace evidente que las madres mantienen una respuesta buena, ya que ellas mayormente acuden a los colegios e instituciones de salud en búsqueda de ayuda que los oriente frente a la enfermedad de su hijo. En tanto que los padres con falta de disponibilidad de tiempo hace que no se involucren en la relación con su hijo y esposa, lo que dificulta la armonía familiar, e influye la actitud entre estos cónyuges.

El padre de familia muchas veces no cuenta con una adecuada información a comparación de la madre, ya que por diversos factores como el trabajo y otras responsabilidades, tiene que permanecer más tiempo en la calle que en la casa (19).

Del 100\% de los padres, según el componente afectivo, se mostró que el 50\% (9) tiene una actitud regular según esta dimensión, y el 50\% (9) es buena. Siendo el 73,91\% (17) madres con una actitud- afectiva regular, en tanto el 26,08\% (6) con una actitud - afectiva buena (Tabla 3).

Si el nacimiento de su niño fue un suceso inesperado, pueda que esto contribuya a un desgaste emocional en ambos padres; sin embargo, algunos emprenderán el acompañamiento con gran ímpetu, pero bajo las características del niño hiperactivo y las expectativas de cambio no vistas tan prontamente como se esperan, puede alterar la conducta tanto de la madre como del padre, quién puede en casos extremos desarrollar sentimientos de rechazo hacia su niño, por lo que existe un grupo de padres y madres que tienen una actitud regular frente a su niño hiperactivo (18).

Lo que puede ser motivo de crisis en una familia quizás no 
lo sea para otra, todo depende de la actitud básica con la que se enfrentan a la vida normalmente y de los mecanismos de adaptación de que se dispongan (4).

La cantidad de afecto que se demuestra a los hijos puede variar de forma considerable, en función de factores culturales y de las diferencias individuales, de personalidad y temperamento, tanto paterna como de los hijos. También varía la forma de expresar ese afecto, su apertura, frecuencia y grado en que se mezcla con rechazo u hostilidad. Los padres descritos como cálidos y protectores suelen sonreír, alabar y animar a sus hijos, limitando sus críticas, castigos y signos de desaprobación. Los padres fríos, hostiles o rechazadores son rápidos en la crítica y el desprecio, castigan o ignoran a sus hijos y limitan sus expresiones de afecto o aprobación (1). Esto quiere decir que los padres muchas veces expresan su disgusto por el niño de forma clara o encubierta, le indican que no es deseado o declaran que cuidarle supone una carga, debido a los síntomas que presentan los niños hiperactivos en su comportamiento.

Si un hijo no se comporta como se supone que deba comportarse, inevitablemente los padres tienden a culparse a sí mismos, o se vuelven muy defensivos porque los demás los culpan por tener un niño ruidoso, que no duerme y es irritable (20).

Los padres de niños con hiperactividad suelen percibir la relación con sus hijos como significativamente más negativa que los padres de niños que no presentan este trastorno. Los problemas que se van sumando llegan a generar en los padres un sentimiento de culpabilidad y fracaso, quienes se consideran ineptos ante la magnitud de problemas que surge diariamente al querer dominar al niño (20).

En esta dimensión afectiva de la actitud puede influir de modo importante el desgaste de los padres, que se va produciendo en la relación entre el niño hiperactivo y sus padres debido al escaso control de los impulsos que presenta el niño, su mal comportamiento, la poca eficacia de los métodos habituales de disciplina y la reiterada frustración que sienten los padres al intentar controlar a su hijo, todo lo cual va generando estrés en la familia y empeorando progresivamente el vínculo entre el niño y sus padres (3). Lo anterior puede desencadenar en que los padres presenten problemas afectivos como depresión, síndrome de cansancio lo cual va influir directamente en la demostración afectiva de los padres hacia los niños, es por ello que se observa dentro de los resultados de investigación que el componente afectivo es regular en los padres.
El involucramiento de ambos padres permite un manejo adecuado en la crianza de sus hijos teniendo efectos muy favorables tanto en el aspecto social y académico del niño hiperactivo, como también en la familia, llegando a fortalecer la relación conyugal, mejorando sus relaciones sociales, permite un buen enriquecimiento del ambiente familiar, a diferencia de padres separados o solteros que puede dificultar el avance del niño, por no tener el apoyo de ambos padres y muchas veces estos presentan una sobrecarga emocional por tener muchas actividades en el trabajo y hogar (21).

La experiencia nos indica que los hombres y las mujeres difieren considerablemente en la manera en que expresan la emoción y en las emociones que eligen para expresar. Con frecuencia las emociones como la simpatía, la tristeza, la empatía y la angustia se consideran «impropias de un hombre» y que los niños son entrenados desde pequeños para inhibir la expresión de estas emociones en público; sin embargo, la actitud de una mujer es comprensiva, atenta, tolerante y amorosa con sus hijos y familiares pero muchas veces estas actitud estereotipadas varían (11).

La actitud del padre de comprensión, tolerancia, y atención, puede verse influida por factores de permanencia con su hijo hiperactivo, no demandando tanto desgaste que la madre, lo que le permite estar más calmado o descansado con respecto a la situación de un niño hiperactivo, por lo que su actitud según el componente afectivo probablemente sea buena, en relación a la madre que es regular; explicado por el cuidado permanente de su hijo y sumada a otras actividades propias de su rol de madre, lo que puede generar una sobrecarga emocional.

Del 100\% (41) de los padres, según el componente conductual, se mostró que el $61,11 \%$ (11) tuvo una actitud buena; el 22,22\% (4) regular, y el 16,6\% (3) mala. El $86,95 \%$ (20) de las madres tuvieron una actitud conductual buena; el 8,69\% (2) tuvo una respuesta regular, y el 4,34\% (1) fue mala.

Las actitudes van a influir en la conducta social, por eso quienes intentan cambiar las conductas de las personas se centran en cambiar las actitudes; por ejemplo, los padres que intentan influir en la conducta de los hijos suelen responder a conductas de aceptación hacia un estilo educativo positivo (2).

Una persona que puede tener una actitud fuertemente negativa (componente afectivo) hacia otra que conoce bien (componente cognitivo) y no obstante relacionarse con ella de forma cordial en una situación dada. Ello se debe a 
que los componentes cognoscitivos y afectivos pertenecen a la esfera privada de la persona, mientras que la conducta es pública y está sujeta a la presión social. El comportamiento no se encuentra únicamente determinado por lo que a las personas les gustaría hacer, sino también por lo que deben hacer. El comportamiento muchas veces está en función de las normas sociales, las cuales han creado hábitos y estereotipos que son los que consolidan una conducta. Existe una compleja red de compromisos sociales que inciden en que la conducta sea, en ocasiones, incongruente con nuestros procesos cognitivos y afectivos. Asimismo, diversos factores hacen que algunas personas actúen más de acuerdo con los componentes cognitivos, emocionales que otras.

Las personas con un nivel alto de autoconocimiento reaccionan frecuentemente más de acuerdo con una situación dada, podría decirse que es una forma de acomodación o adaptación social a las circunstancias. Sin embargo, las personas de bajo autoconocimiento actúan, en general, de forma congruente con los procesos cognitivos/afectivos, manifestando abiertamente a través de su conducta las actitudes que poseen ante determinada situación, alegría, enojo, dolor, impaciencia, etc. (9).

Las actitudes forman parte e integran la personalidad del sujeto, son hábitos internos que indican las tendencias reales de la conducta manifiesta, la cual representa la mejor información para predecir un comportamiento, a diferencia de las expresiones verbales de las ideas, opiniones o imágenes mentales que, en ocasiones, no se corresponden con las conductas o acciones de las personas, tal como refieren Secord y Backman: Lo que tú eres, resuena con tanta fuerza en mis oídos que no puedo escuchar lo que dices (7).

Las madres presentan una buena actitud en la dimensión conductual, posiblemente porque al permanecer en el hogar y cuidar en forma permanente a los niños, desarrollan algunos mecanismos que les permite tener un afrontamiento positivo, teniendo mayor tolerancia hacia el comportamiento de sus hijos, en cambio el padre no se encuentra en el hogar, por motivo de trabajo, ya que él es el sustento económico y busca el bienestar de su familia y cuando llega a su hogar, el niño lo satura con el comportamiento que presenta, no reconociendo el comportamiento que presenta el niño hiperactivo en la diferentes situaciones en que se va encontrar.

Los padres y madres ofrecen a sus hijos hiperactivos la oportunidad de desarrollarse en un sentido individualizado y no como una copia de los padres o como a ellos les gustaría que lo hicieran. La autodeterminación de cada padre y madre responde a cada habilidad individual conductual a desarrollar en su hijo hiperactivo. Los padres/madres proveen al niño de habilidades sociales, sino en cómo interactuar con otros niños, respetar sus derechos y la intimidad de los otros y en cómo vivir con éxito en la sociedad.

Un reforzador puede ser cualquier cosa que incremente la probabilidad de dar una actitud buena en mayor proporción en relación con la actitud regular y baja; por consiguiente el elogio, la comida, una sonrisa son reforzadores hacia conductas positivas mostradas por los padres y madres de niños hiperactivos.

La familia es el espacio en que los niños realizan sus primeras experiencias de interacción. La alianza familiar se organiza entre el niño y sus cuidadores primarios. Los cuidadores facilitan y guían el desarrollo del niño, mientras este crece y logra su autonomía. La familia cumple la función de asegurar la supervivencia del niño, y el crecimiento y desarrollo adecuados para luego facilitar la salida del núcleo primario armando nuevos vínculos.

«Madre» y «padre» son funciones, más allá de los seres humanos que las encarnen, y el buen desarrollo de un niño dependerá de la complementariedad de estas funciones (22).

\section{REFERENCIAS BIBLIOGRÁFICAS}

1. Filomeno A. El Trastorno por Déficit de Atención con Hiperactividad en las últimas cuatro décadas y media en el Perú.: Problemas actuales y búsqueda de soluciones. RevMedHered. [Internet]. jul./set. 2006 [citado 26 Ene 2012];17(3):[aprox. 3p.]. Disponible en: $<$ http://www.scielo.org.pe/scielo.php?script $=$ sci_arttext\&pid=S $1018-130$ X200600030 $0001 \& \operatorname{lng}=$ es\&nrm=iso $>$. ISSN 1018-130X.

2. García de la Espriella MC, Cervantes MA. Efectos de un ambiente virtual de apoyo a la enseñanza tradicional en la actitud de los alumnos hacia la resolución de problemas matemáticos [tesis]. Barranquilla: Universidad del Norte;2012.

3. Santa Cruz De León, E.; Pérez Villalba, E. y Palacios Muñoz, U.: «Análisis Cualitativo de la competitividad de las empresas frutícolas del soconusco, Chiapas» en observatorio de la Economía Latinoamericana, $\mathrm{N}^{\circ}$ 169, 2012. Disponible en: http://www.eumed.net/ curseoon/ecolat/mx/2012.

4. Sabate N, Basses N, Quiles I. Trastorno por déficit de atención. En: Tomas J. (Ed). Actitudes educativas, 
Trastornos del lenguaje y otras alteraciones en la infancia y adolescencia. Barcelona: Alertes; 1999. p.190-202.

5. Ministerio de Salud. Guía práctica para consejería para Adolescentes y Jóvenes. Gobierno de Chile; 2011.

6. Menal PB. El alumno con TDAH-trastorno de déficit de atención con o sin hiperactividad.2da edición. Barcelona: Mayo; 2006.

7. Miranda-Casas A, Grau-Sevilla D, Marco-Taberner R. Rosello B. Estilos de disciplina en familias con hijos con trastorno por déficit de atención/hiperactividad: influencia de la evolución del trastorno. Revista de Neurología [Internet]. 2007 [citado 3 de junio de 2011].44(2):S23-S25.

8. Watson RI, Lindaren HC. Psicología del niño y adolescente. 4a ed. México, D.F.: Limusa; 2001.

9. Wick NR. Sicopatología del niño y del adolescente. 3a ed. Madrid: Prentice Hall; 2002.

10. Flores AC, Montenegro AM. Factores de riesgo biológicos asociados a niños con trastorno por déficit de atención e hiperactividad [tesis]. Lima: Universidad Nacional Mayor de San Marcos; 2002.

11. Miranda-Casas A, Grau-Sevilla D, Marco-Taberner R, Roselló B. Estilos de disciplina en familias con hijos con trastorno por déficit de atención/hiperactividad. Influencia en la evolución del trastorno. REV NEUROL 2007;44 (Sup1 2): S23-S25.

12. Asociación Peruana de Deficit de Atención [Internet] Lima: La Asociación; c2003-2011 [actualizado 17 May 2012; [citado 13 junio 2011]. APDA; [aprox. 3p.]. Disponible en: http://deficitdeatencionperu.com/ category/boletines.

13. Castro J. Psiquiatría infantil. En: Castro J, editor. Niñas, niños y adolescentes: exclusión y desarrollo psicosocial. Lima: IFESANT; 2001. p. 50-60.
14. Caballo, E, Simón MA, (Ed). Manual de psicología clínica infantil y del adolescente: transtornos generales. Madrid: Pirámide; 2001.

15. Marriner Torney A. Modelos y teorías de enfermería.6ta ed. Madrid: Elservier Mosby; 2007.

16. Miguel MD. Formación de las actitudes y proceso de cambio. En: Novel G, editor. Enfermería psicosocial y salud mental. Barcelona: Masson; 2005. p. 31-35.

17. MorrisChG, Maisto AA (Ed). Introducción a la Psicología. 9na ed. México: Pearsons Education; 2005.

18. Papalia DE, Wendkos S. Psicología del desarrollo. 7ma ed. Bogotá: McGraw Hill; 1998.

19. Watson RI, Lindaren HC. Psicología del niño y adolescente. 4a ed. México, D.F.: Limusa; 2001.

20. Ollendick TH, Versen M. Psicología infantil. Barcelona: Martínez Roca; 1998.

21. Hauser P. Trastorno por déficit de atención con hiperactividad. Washington D.C.: American PsychiatricPress; 2005.

22. Armus M, Duhalde C, Oliver M, Woscoboinik N. Desarrollo emocional. Clave para la primera infancia. Fondo de las Naciones Unidas para la Infancia (UNICEF). 1ra. Ed. Buenos Aires: Fundación Kaleidos; 2012.

\section{Correspondencia}

Zulema Silva Mathews

Universidad Peruana Cayetano Heredia

Dirección: Miguel Baquero 251 - Lima

Correo electrónico: zulema.silva@upch.pe

Forma de citar este artículo: Mendoza-Reyes $P$, MontesinosMatos P, Valderrama-Mendoza V, Silva- Mathews Z. Actitud de los padres frente al comportamiento de sus hijos hiperactivos. Rev enferm Herediana. 2012;5(2):97-104. 